\title{
Transaction
}

\section{Near-Infrared Green Camouflage of PET Fabrics Using Disperse Dyes}

\author{
Zhang $\mathrm{Hui}^{* 1}$ and Zhang Jianchun ${ }^{* 2}$ \\ ${ }^{* 1}$ School of Textile \& Materials, Xi' an Polytechnic University, XI' an 710048, People's Republic of China \\ ${ }^{* 2}$ The Quartermaster Research Institute of General Logistics Department of the CPLA, Beijing 100088, \\ People's Republic of China
}

\begin{abstract}
In order to match the reflectance profile of greenish leaf at NIR region, four commercially disperse dyes were used to dye PET fabric, and the reflectance of dyed fabric and the transmittance of dye liquor in alcohol solution were measured by using a UV-Vis-NIR spectrometer with an integrating sphere. The effect of the combination dyeing, dyeing concentration (\% owf) and fabric weave on the reflectance were also studied. The results show that the reflectance of the combination dyeing is determined by one of the dyes whose reflectance curve emerges at longer wavelengths. C.I. Disperse Blue 56 plays an important role in green camouflaging. When the dyeing concentration of C.I. Disperse Blue 56 is $1-2 \%$ owf, the reflectance of dyed fabric almost overlaps that of greenish leaf in red shift region (region $A$ ). Fabric weave has little effect on the reflectance for the same dyeing program.
\end{abstract}

(Received 6 January, 2007 ; Accepted 26 June, 2007)

\section{Introduction}

From a defensive standpoint, there is constant research to find sophisticated means of deceiving the military observer, i.e. to make personnel less visible, whether in daylight or by night. Concealment from daylight visual surveillance is a long-established practice in which different hues are employed to overcome the contrast of an object and its surroundings [1]. The hues found in most camouflage patterns are green, olive, khaki, brown and black. However, with the advent of NIR surveillance equipments, such as night-vision devices, the image intensifier and NIR photography, it became necessary to take into account the IR reflectance of paints and garments in the NIR region (700 - $1300 \mathrm{~nm})$ [2-3]. It therefore became necessary for camouflage patterns to be painted or printed with selected dyes and pigments having NIR reflectance that matched the expected surrounding topography [4]. For military personnel it is necessary to wear the appropriate clothing that attempts to minimize the contrast between objects and their environment over the NIR region in addition to the visible region. Results show that the NIR camouflage properties of dyed fabric have relations with the constitution of dyes to a great extent. Different fabrics can be dyed by using a variety of dyes. The same fabric can also be dyed by using different dyes to claim the requirement of NIR camouflage [5]. Given the sensitive nature of research into NIR camouflage, it is unsurprising that much of the work on the synthesis, formulation and application of colorants remains confidential.

Despite the fact that cotton can be dyed in camouflage colors by using existing technology, conventional methods for the coloration of other fibres have been found inadequate [6]. In this paper, the reflectance profiles of different greenish leaves were measured at first, and then PET and PET/cotton fabrics were dyed by using four disperse dyes to resemble the characteristics of greenish leaves. The reflectance of dyed fabric in NIR region was studied. The effect of dyeing concentration and fabric weave on the reflectance was also investigated.

\section{The reflectance characteristics of greenish leaves}

It is obvious that the reflectance curve of different vegetation varies a little in the visible and NIR wavebands. Even for the same vegetation, the reflectances are also different during its growing phase [3]. Hence, the typical reflectance profile of greenish foliage (chlorophyll) is generally selected as a standard reference which we called the standard reflectance curve. We chose different species of fresh greenish leaves and measured their reflectance curves, as illustrated in Figure 1. Only three reflectance curves are presented (the deciduous tree leaf comes from Populus tomentosa, the coniferous needle belongs to Cedrus deodara), whereas 
other reflectance curves are within the scope of them. It can be seen that the reflectance curves have a characteristic shape bestowed by chlorophyll. They have a small increase in reflectance at around $540 \mathrm{~nm}$, which is responsible for the green coloration. Reflectance then decreases slightly in the red region and rises steeply between 670 and $760 \mathrm{~nm}$ to a plateau at $780 \mathrm{~nm}$, here we call it (670 - $780 \mathrm{~nm}$ waveband) the reflection red shift region (region $A$ ). At longer wavelengths the reflectance remains fairly constant until about $1300 \mathrm{~nm}$, where it decreases again, here we call it (780 - $1300 \mathrm{~nm}$ waveband) the reflection luminance region (region $B$ ). The level of the plateau is dependent on vegetation type : deciduous tree leaves have relatively high IR reflectance (about $85 \%$ ), whereas coniferous needles are inclined to be more strongly absorbing in the infrared (the reflectance is about $50 \%$ ). Beyond $1300 \mathrm{~nm}$, there are two absorption regions around $1400 \mathrm{~nm}$ and $1900 \mathrm{~nm}$, which correspond to the water. The reflectances of both sides for the same leaf are also different. The reflectance of upper side is larger than that of down side. Therefore, in order to obtain NIR green camouflage for PET fabric, the reflectance curve of dyed fabric must not only overlap in region $A$, but also locate in region $B$.

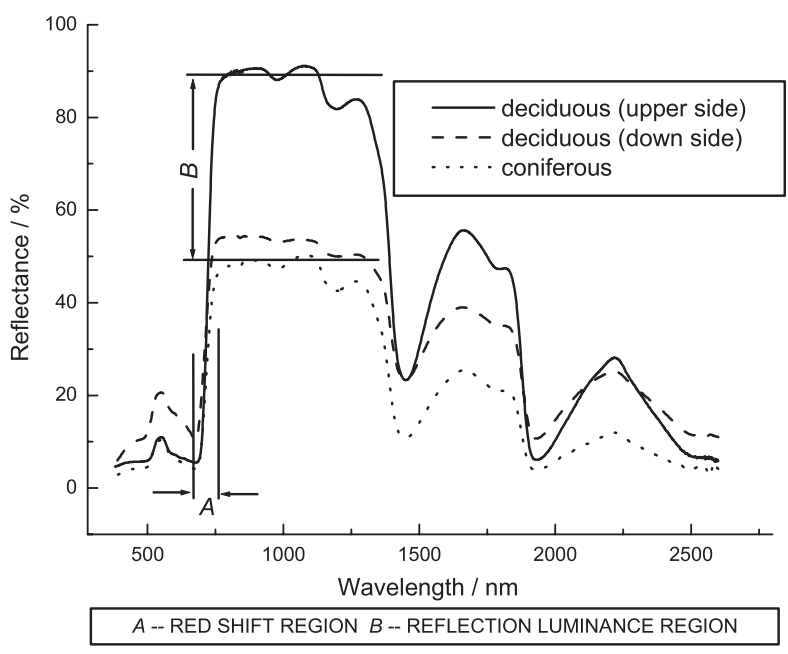

Fig. 1 Reflectance curves of greenish leaves.

\section{Experimental}

\subsection{Materials}

The dyeing experiments were carried out on five different fabrics. Commercially-obtained PET fabric (sample 1\#) was scoured with a solution of $1 \mathrm{~g} / 1$ sodium hydroxide, $4 \mathrm{~g} / 1$ soap and $0.3 \mathrm{~g} / 1$ sodium silicate for 60 min at $95{ }^{\circ} \mathrm{C}$, and then rinsed with deionised water and dried at room temperature. Prepared PET/Cotton (65/35) plain fabric (2\#) was provided by local dyeing plant. We fabricated three PET fabrics (3\# - 5\#) on a SL7900 sampling loom (CCI Tech Inc.) with 100D/48f filaments, and treated them with the same method above mentioned. All tested sample features are given in Table 1.

Disperse dyes of low energy level used in the experimental are given in Table 2, such as C.I. Disperse Black (C.I.UNK) $100 \%$, C.I. Disperse Yellow 23 (C.I.26070) 100 \%, C.I. Disperse Blue 14 (C.I.61500) 100 $\%$ and C.I. Disperse Blue 56 (C.I.63285) $100 \%$, which were provided by Dystar Trading Co. Ltd. C.I. Disperse Black is composed of C.I. Disperse Orange 30 (C. I.11119), C.I. Disperse Red 54 (C.I.UNK) and C.I. Disperse Blue $79: 1$ (C.I.11344) in a certain proportion. Acetic acid and sodium hydrosulfite were of analytical grade. The dispersing agent $N N O$ was obtained from local dyeing plant.

\subsection{Dyeing method}

Dyeing trials were carried out in a laboratory-type dyeing machine (IR-12P DYER) made by Taiwan LABORTEX Co. Ltd. at a liquor-to-goods ratio of $50: 1$. The weight of each sample was $2 \mathrm{~g}$ and a total dye bath volume was $100 \mathrm{ml}$. The $\mathrm{pH}$ was adjusted to 5.5 by $1 \mathrm{~g} / \mathrm{l}$ acetic acid and $1 \mathrm{~g} / 1$ of the dispersing agent $N N O$, which also has a leveling action, and was used in the dye baths. The temperature program for the disperse dyestuffs is shown in Fig. 2. The combination of dyes and dyeing concentration for different fabrics are given in Table 3 .

The dyed fabrics were washed in a solution containing $2 \mathrm{~g} / 1$ sodium hydrosulfite and $2 \mathrm{~g} / 1$ sodium hydroxide at $70{ }^{\circ} \mathrm{C}$ for $15 \mathrm{~min}$ at a liquor ratio of $80: 1$. The fabric samples were then rinsed in hot and cold water and left to be dried under ambient conditions.

The dyeing experiments were performed in duplicate. Two samples were separately dyed and color measurements were performed on these samples. Three reflection measurements were made on each sample rotating the samples $90^{\circ}$ before each measurement. The average of the measurements of the two samples was recorded as reflectance and used in calculating the $K / S$ values. These reflectance curves were used to calculate the CIE1976 L*, a* and $b^{*}$ color coordinates. Comparison of the color coordinates of green samples with the standard greenish leaf allowed calculation of the variability of the color of dyed fabric in the form of the CIELAB color difference $(\Delta E)$.

The $K / S$ values of each dyed sample at the maximum light absorption wavelength were measured by using a Datacolor SF600 Plus colorimeter. The reflectance measurements of each green sample were performed between 400 and $700 \mathrm{~nm}$ under D65/10 
Table 1 Parameters of fabric.

\begin{tabular}{|c|c|c|c|c|c|c|}
\hline \multirow{2}{*}{ Sample } & \multirow{2}{*}{ Material } & \multirow{2}{*}{ Fabric weave } & \multicolumn{2}{|c|}{ Linear density, Tex } & \multicolumn{2}{|c|}{ Density, thread $/ 10 \mathrm{~cm}$} \\
\hline & & & Warp & Weft & Warp & Weft \\
\hline $1 \#$ & PET & 2/1 twill weave & 32.0 & 32.0 & 278 & 232 \\
\hline $2 \#$ & PET/cotton & Plain weave & 30.0 & 30.0 & 340 & 245 \\
\hline $3 \#$ & PET & Plain weave & 11.1 & 11.1 & 280 & 250 \\
\hline $4 \#$ & PET & $\begin{array}{c}\text { 8-harness satin } \\
\text { weave }\end{array}$ & 11.1 & 11.1 & 402 & 380 \\
\hline $5 \#$ & PET & Honeycomb weave & 11.1 & 11.1 & 406 & 384 \\
\hline
\end{tabular}

Table 2 Disperse dyes used in the dyeing experiments.

\begin{tabular}{cccc}
\hline Energy Level & C.I. No. & Wavelength of max. Abs. & Chemical class \\
\hline Low & C.I. Disperse Black (C.I.UNK) & $510 \mathrm{~nm}$ & Monoazo \\
Low & C.I. Disperse Yellow 23 & -- & Azo \\
& (C.I.26070) & & \\
Low & C.I. Disperse Blue 14 (C.I.61500) & $615 \mathrm{~nm}$ & Antraquinone \\
Low & C.I. Disperse Blue 56 (C.I.63285) & $550 \mathrm{~nm}$ & Antraquinone \\
\hline
\end{tabular}

Table 3 Dyeing formula.

\begin{tabular}{ccc}
\hline No. & Dyeing sample & Combination of dyes and dyeing concentration (owf) \\
\hline 1 & $1 \#$ & C.I. Disperse Blue $14,1 \%$ \\
2 & $1 \#$ & C.I. Disperse Blue $56,1 \%$ \\
3 & $1 \#$ & C.I. Disperse Yellow $23,1 \%$ \\
4 & $1 \#$ & C.I. Disperse Black, $1 \%$ \\
5 & $1 \#$ & C.I. Disperse Blue $14,1 \%$; C.I. Disperse Blue $56,1 \% ;$ \\
6 & $1 \#$ & C.I. Disperse Yellow, $2 \% ;$ and C.I. Disperse Black, $0.2 \%$ \\
7 & $1 \# ; 2 \#$ & C.I. Disperse Blue 56, 0.05; 0.1; 0.3; 0.5; $1 ; 1.5 ; 2 ; 3 ; 4 ; 5 ; 6 ; 7 \%$ \\
8 & $3 \#-5 \#$ & and C.I. Disperse Black, $0.05 \%$ \\
\hline
\end{tabular}

illuminant by using a Datacolor SF600 Plus colorimeter using a measuring area with a diameter of $9 \mathrm{~mm}$. The reflectance profiles in 380 - $2600 \mathrm{~nm}$ waveband were

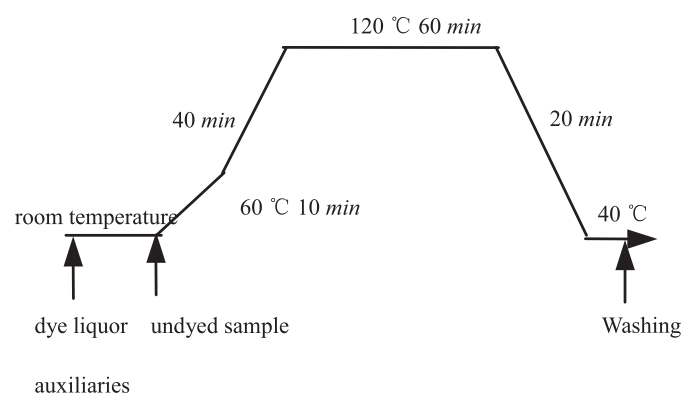

Fig. 2 Temperature program for the dyeing of samples. measured by using a UV-Visible-NIR spectrophotometer U-4100 (Hitachi co. Ltd., Scan speed : $1200 \mathrm{~nm} / \mathrm{min}$ at visible range, $1500 \mathrm{~nm} / \mathrm{min}$ at NIR range) with an integrating sphere (ø60 mm).

\section{Results and discussion}

\subsection{Reflectance of the combination dyeing}

The reflectance of the combination dyeing had relation to the constitution of dyes at NIR region. The reflectance curves using dyeing formula No.1, NO.2, No.3, NO.4 and No.5 (see Table 3) are given in Fig. 3. It can be seen that the reflectance of dyed fabric (No.3) using C.I. Disperse Yellow 23 increases sharply in the 
range $400-600 \mathrm{~nm}$. The reflectances of C.I. Disperse Blue 56 (No.2), C.I. Disperse Black (No.4) and C.I. Disperse Blue 14 (No.1) increase rapidly in the corresponding range $630-800 \mathrm{~nm}, 470-820 \mathrm{~nm}$ and $660-880 \mathrm{~nm}$, respectively. However, the reflectance of combination dyeing (No.5) increases in the $630-880 \mathrm{~nm}$ waveband, namely, the absorption edge almost overlaps that of C.I. Disperse Blue 14. This indicates that the reflectance curve of combination dyeing at NIR region is determined by one of the dyes whose reflectance emerges at longer wavelengths. This conclusion can be extended to other disperse dyes in our experiments. It is obvious that the reflectance of C.I. Disperse Blue 56 is very close to that of the greenish leaves at region $A$. So C.I. Disperse Blue 56 plays an important role in NIR camouflage.

\subsection{Reflectance of PET fabric before and after dyeing}

The reflectance curves of PET fabric (sample 1\#) using dyeing formula No.2, No.3 and No.4 are presented in Fig. 4. The transmission curves of dye liquors in alcohol solution are also given. The absorption peak of undyed fabric occurs at $408 \mathrm{~nm}$. It is seen from Fig. 4(a) that there is no typical absorption peak for C.I. Disperse Yellow 23 in visible range, and the wavelength at absorption peak (408 nm) after dyeing does not change. It is obvious that the reflectances of dyed fabric from 408 to $594 \mathrm{~nm}$ decrease rapidly partly due to the dyeing of fibres, meanwhile, it is also influenced by the reflectance of the fibre itself. The absorption peak of C.I. Disperse Black is at $510 \mathrm{~nm}$, but the corresponding absorption peak of dyed fabric shifts to shorter wavelength, at $468 \mathrm{~nm}$, as illustrated in Fig. 4(b). The absorption peak of C.I. Disperse Blue 56 is at $550 \mathrm{~nm}$, and the corresponding absorption peak of dyed fabric is within the range of 576 $640 \mathrm{~nm}$ shown in Fig. 4(c). Hence, the reflectance of dyed

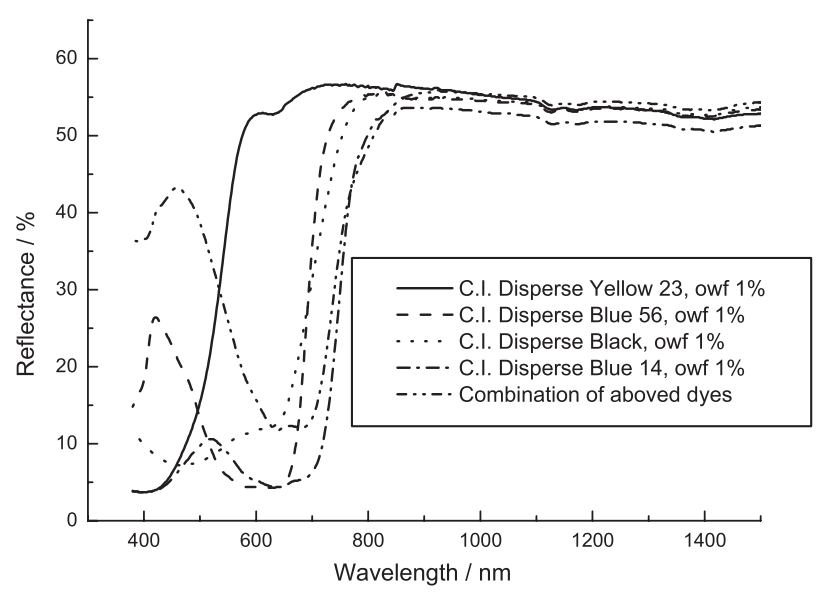

Fig. 3 Reflectance curves of dyed fabrics using single dye and combination dyeing. fabric depends markedly upon the characteristics of dyes in visible range. Although C.I. Disperse Blue 56 (1,5-di $\mathrm{NH}_{2}$-4,8-di OH-2Br-anthraquinonoid) and C.I. Disperse Blue 14 (1,4-di $\mathrm{OH}$ anthraquinonoid, the measured absorption peak at $615 \mathrm{~nm}$ ) are anthraquinonoid dyes, their absorption peaks occur at $552 \mathrm{~nm}$ and $619 \mathrm{~nm}$, respectively [7], which accord with the results we measured. This is due to the fact that the absorption maxima in visible range are determined by the electrondonating power of the substitutes, that is to say, 1,4-disubstituted anthraquinones absorb at much longer wavelengths than 1,5- and 4,8-disubstituted derivatives.

With regard to NIR waveband, the wavelengths of typical absorption peaks don't shift after dyeing, but the reflectances decrease to some extent. It is seen from Fig. 4 that the absorption peaks of three dye liquors at $974 \mathrm{~nm}$, $1194 \mathrm{~nm}$ are attributed to hydroxy group and methyl group (C-H stretching 2nd overtone), respectively. The infrared is absolutely absorbed by dye liquor beyond $1380 \mathrm{~nm}$. The other bond vibrations of PET fibre are given as follows: $1170 \mathrm{~nm}$ (C-H stretching 2nd overtone), $1410 \mathrm{~nm}$ (O-H stretching 1st overtone), 1660 $\mathrm{nm}$ (C-H stretching 1st overtone), $1906 \mathrm{~nm} \mathrm{(O-H}$ stretching $1 \mathrm{st}$ overtone), $2134 \mathrm{~nm}(\mathrm{~N}-\mathrm{H}$ and $\mathrm{C}=\mathrm{O}$ stretching), $2254 \mathrm{~nm} \quad(\mathrm{O}-\mathrm{H}$ stretching and $\mathrm{O}-\mathrm{H}$ deformation), $2332 \mathrm{~nm}$ (C-H stretching and C-H deformation).

\subsection{Effect of dyeing concentration on reflectance}

We chose dyeing formula No.6 to study the effect of dyeing concentration on reflectance. Fig. 5 presents the reflectance curves using C.I. Disperse Blue 56 at different dyeing concentrations. They are very similar to each other in shape. The more the dye concentration in the fibre, the less the proportion of reflection [8]. The wavelength at absorption peak $(630 \mathrm{~nm})$ does not shift. As the dyeing concentration increases, the reflectance decreases gradually, but the reflectance turn flat around $630 \mathrm{~nm}$. The absorption edge in region $A$ overlaps that of greenish leaves on condition that the dyeing concentration is between $1 \%$ and $2 \%$ owf. The average reflectance at region $B$ is about $66 \%$. So the suitable dyeing concentrations of C.I. Disperse Blue 56 for NIR camouflage are $1 \%$ owf for dyeing light color and $2 \%$ owf for dyeing dark color.

\subsection{Effect of blend fabric on reflectance}

The reflectance curves are given in Fig. 6 by employing dyeing formula No.7. It is evident that the reflectance of PET/cotton fabric (sample 2\#) is relatively higher than that of PET fabric (sample 1\#) for the same dyeing formula (about $8 \%$ ) because of the undyed cotton 
fibres. This indicates that the reflectance in region $B$ can be improved to some extent by blending different fibres, which have stronger reflection ability. Furthermore, the wavelength at absorption peak does not change. The typical absorption peaks of cotton are at $1486 \mathrm{~nm}(\mathrm{C}-\mathrm{H}$ stretching 1nd overtone), $1810 \mathrm{~nm}$ (O-H stretching and C-O stretching 1st overtone), $2254 \mathrm{~nm}$ (O-H stretching and $\mathrm{O}-\mathrm{H}$ deformation), $2336 \mathrm{~nm}$ (C-H stretching and $\mathrm{C}-\mathrm{H}$ deformation), respectively.

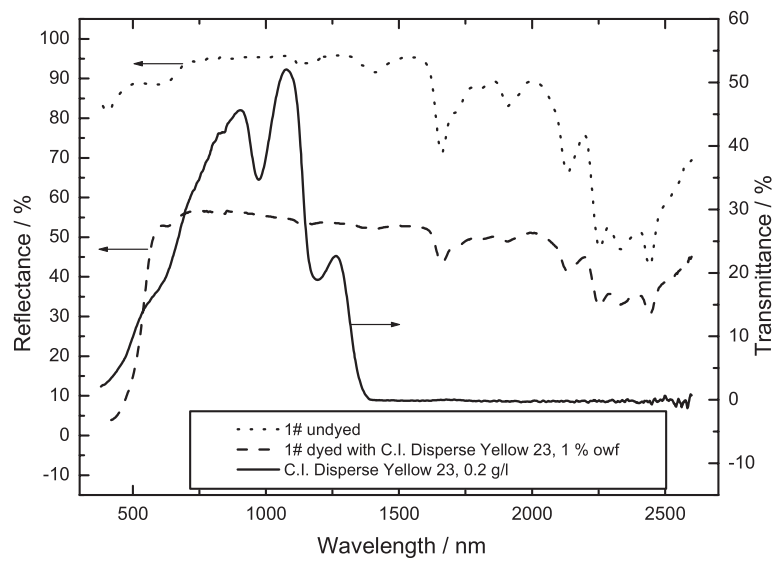

(a) C.I. Disperse Yellow 23

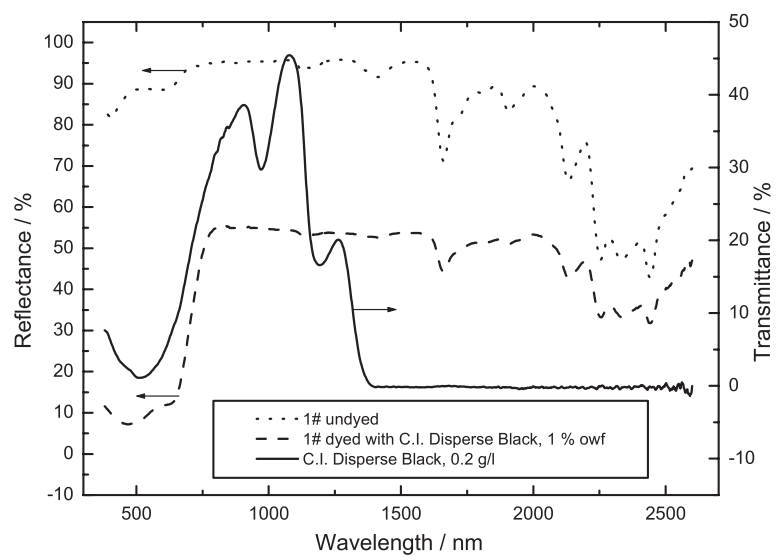

(b) C.I. Disperse Black

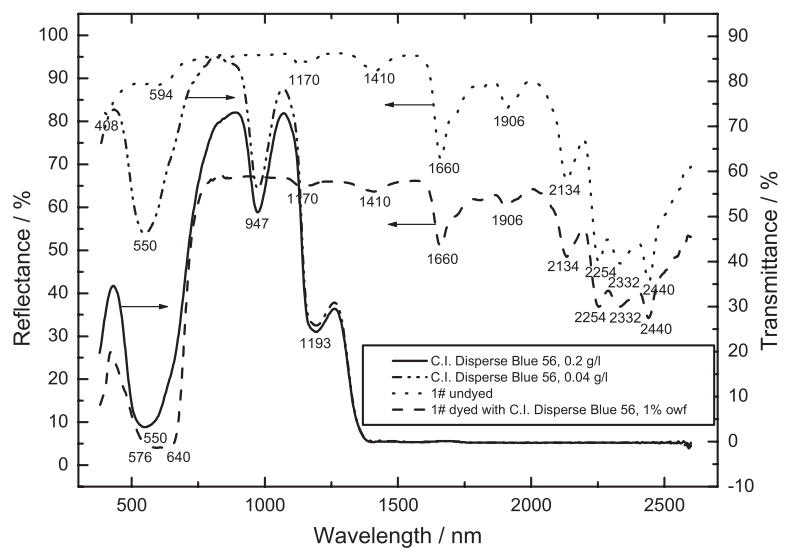

(c) C.I. Disperse Blue 56

Fig. 4 Transmittance curves of dye liquors and reflectance profiles of dyed fabrics.

\subsection{Effect of fabric weave on reflectance}

In order to investigate the effect of fabric weave on reflectance, we fabricated three PET fabrics, and then dyed them using the same dyeing formula No.8. The reflectance curves are presented in Fig. 7. The reflectances of plain fabric (3\#), 8-harness satin fabric

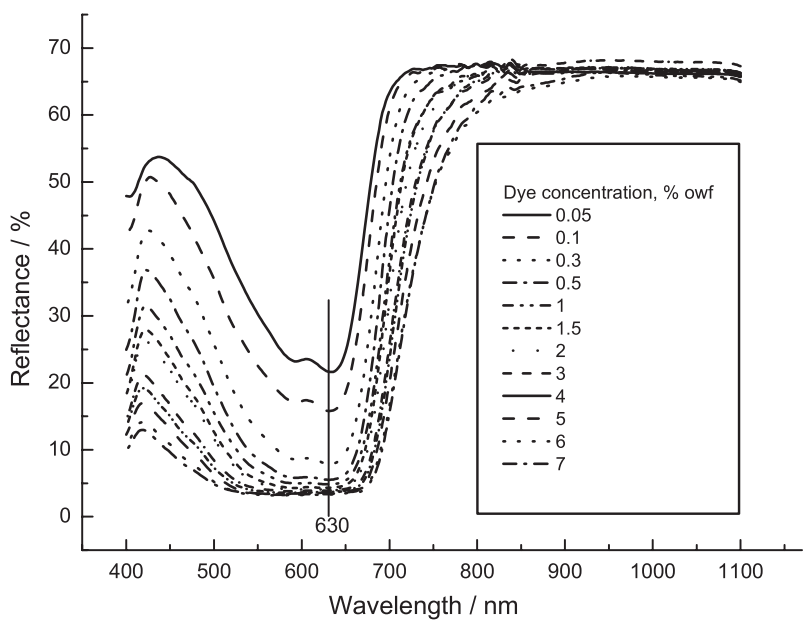

Fig. 5 Reflectance curves at different dyeing concentrations.

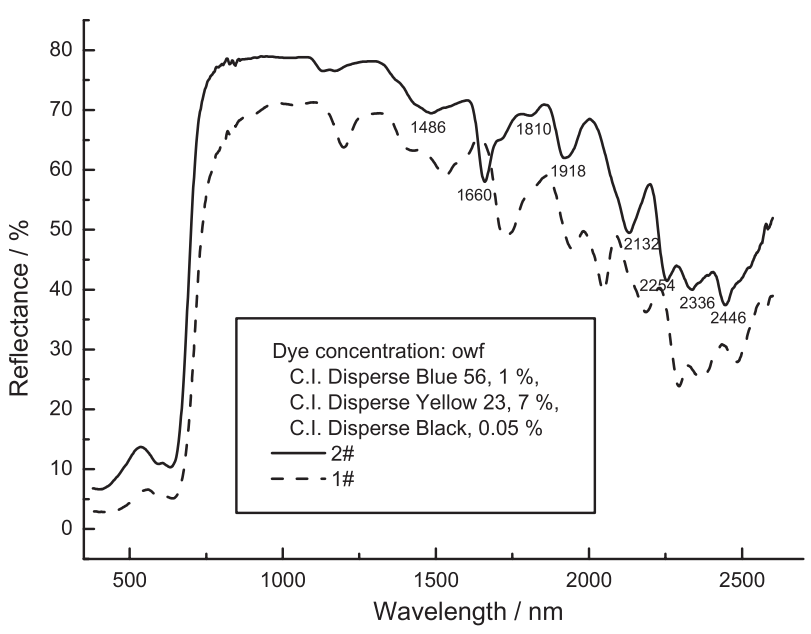

Fig. 6 Reflectance curves of PET/cotton and PET fabrics.

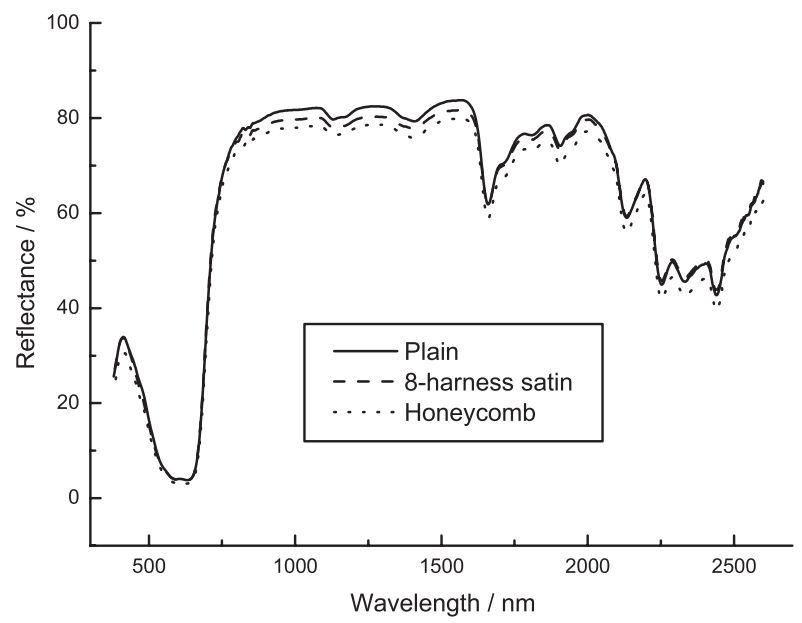

Fig. 7 Reflectance curves of different weave fabrics. 
Table 4 Color coordinates of dark and pale green fabric relative to the greenish leaf of white birch.

\begin{tabular}{cccccccc}
\hline PET sample & $\mathrm{L}^{*}$ & $\mathrm{a}^{*}$ & $\mathrm{~b}^{*}$ & $\Delta \mathrm{L}^{*}$ & $\Delta \mathrm{c}^{*}$ & $\Delta \mathrm{H}^{*}$ & $\Delta \mathrm{E}^{*}$ \\
\hline Dark green & 28.22 & -4.75 & 3.80 & 0.23 & 0.67 & -1.47 & 1.63 \\
Pale green & 28.04 & -3.35 & 4.36 & 0.05 & 0.09 & -0.08 & 0.13 \\
\hline
\end{tabular}

(4\#) and honeycomb weave fabric (5\#) almost overlap in visible wavebands. There is a little discrepancy in NIR region, about $3 \%$. This suggests that the fabric surface has no effect on visible and NIR camouflage.

\subsection{Reality of visible and NIR camouflage}

Fig. 8 shows the reflectance curves of dark and pale green PET fabrics using C.I. Disperse Blue 56, C.I. Disperse Yellow 23 and C.I. Disperse Black. The reflectances are very close to each other, and almost overlap 'that of greenish leave in region $A$. Meanwhile, they are located between deciduous tree leaf and coniferous needle in region $B$, which meet the requirement of NIR camouflage. We also found that the dyeing concentration of C.I. Disperse Black has a great effect on NIR camouflage beyond $0.5 \%$ owf.

In order to verify the effect of visible camouflage, we measured the chromatic values (CIE1976 L", a* and b*) of pale and dark green fabrics, and then calculated the color differences between green PET fabrics and the greenish leaf of white birch (Betula platyphylla, $\mathrm{L}^{*}=$ $\left.27.99, a^{*}=-3.23, b^{*}=4.34\right)$. The results are presented in Table 4. This indicates that green PET fabrics are able to simulate greenish leaves.

\section{Conclusions}

Based on NIR camouflage theory, PET fabrics were dyed by using a combination of dyes (C.I. Disperse Blue

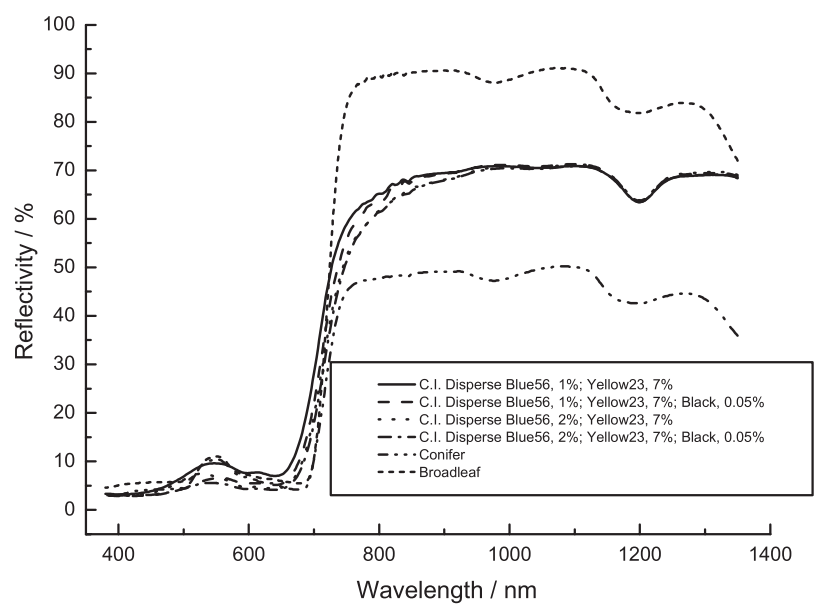

Fig. 8 Reflectance curves of green PET fabrics.
56, C.I. Disperse Yellow 23 and C.I. Disperse Black) to imitate the reflectance profile of greenish leaf. Results show the absorption edge is determined by one of the dyes whose reflectance curve emerges at longer wavelengths. C.I. Disperse Blue 56 plays an important role in NIR green camouflage. When the dyeing concentration of C.I. Disperse Blue 56 is 1 - $2 \%$ owf, the reflectance of dyed fabric matches with that of greenish leaf in region $A$. Fabric weave has no effect on the reflectance for the same dyeing formula. For PET/Cotton blended fabric, the reflectance of PET/cotton fabric is larger than that of PET fabric on condition that PET fibres were dyed.

\section{Acknowledgements}

The research reported in this paper was funded by Natural Science Research Plan (No.06JK299) from Shaanxi Education Department in China, Scientific and Technological Project (No.2004K08-G17) from Shaanxi Science \& Technology Department in China. The work was also supported by a Scientific and Technological Project (No.GG04055) from Xi'an Science Technology Bureau in China.

\section{References}

1. J. Ch. Zhang, "Patten Painting Camouflage Technology", China Textile Industry Press, Beijing, p.60 (2002).

2. Z. F. Wu, M. L. Liu, Sh. J. Zhang, Y. Z. Zhao, and H. B. Li, "Infrared and Twilight Technology", National Defence Industry Press, Beijing, p.3 (1998).

3. Y. Zh. Wang, "Modern Military Optical Technology”, Science Press, Beijing, p.59 (2003).

4. K. K. Gupta, A. Nishkam, and N. Kasturiya, Journal of Industrial Textiles, 31, 27 (2001).

5. R. A. Coleman, Technical report (ADA000832), Chemical Research Division, Cyanamid Co., New Jersey, USA (1974).

6. S. M. Burkinshaw, G. Hallas, and A. D. Towns, Rev. Prog. Coloration, 26, 47 (1996). 
7. Yoshiya Kogo, Hikaru Kikuchi, Masaru Matsuoka, and Teijiro Kitao, JSDC, 96, 475 (1980).
8. J. Z. Yang, "Analysis and Anatomy of Dyes", Chemistry Industry Press, Beijing, p.141 (1987). 